\title{
Transparency and perfect absorption of all-dielectric resonant metasurfaces governed by the transverse Kerker effect
}

\author{
Hadi K. Shamkhi, ${ }^{1}$ Andrey Sayanskiy, ${ }^{1}$ Adrià Canós Valero, ${ }^{1}$ Anton S. Kupriianov, ${ }^{2}$ Polina Kapitanova, ${ }^{1}$ \\ Yuri S. Kivshar, ${ }^{3}$ Alexander S. Shalin, ${ }^{1}$ and Vladimir R. Tuz $\mathbb{B}^{4,5}$ \\ ${ }^{1}$ ITMO University, St. Petersburg 197101, Russia \\ ${ }^{2}$ College of Physics, Jilin University, Changchun 130012, China \\ ${ }^{3}$ Nonlinear Physics Centre, Australian National University, Canberra ACT 2601, Australia \\ ${ }^{4}$ International Center of Future Science, State Key Laboratory of Integrated Optoelectronics, \\ College of Electronic Science and Engineering, Jilin University, Changchun 130012, China \\ ${ }^{5}$ Institute of Radio Astronomy, National Academy of Sciences of Ukraine, Kharkiv 61002, Ukraine
}

(Received 27 May 2019; published 19 August 2019)

\begin{abstract}
Dielectric metasurfaces allow us to realize many unique effects in optics, and they can serve as the building blocks of the modern photonic technologies. Here, we suggest theoretically and demonstrate experimentally the effect of high transparency of all-dielectric metasurfaces with meta-atoms supporting the so-called transverse Kerker effect. In contrast to the well-known Huygens' metasurfaces, in our case both phase and amplitude of the incoming wave remain unperturbed at the resonant frequency and, consequently, our novel metasurfaces totally operate in the high-transparency regime. We prove experimentally, in the microwave frequency range, that both phase and amplitude of the waves transmitted through these metasurfaces remain almost unaffected. Also, we demonstrate numerically and experimentally and explain theoretically a novel mechanism for achieving a perfect absorption of the incident light enabled by the resonant response of the dielectric metasurfaces placed on a conducting substrate. In the subdiffractive limit, we show that these effects are mainly determined by the optical response of the constituting meta-atoms rather than collective lattice contributions. With the spectrum scalability, our finding can be extended to the optical frequencies to be employed for energy harvesting, nonlinear phenomena, and filtering of light.
\end{abstract}

DOI: 10.1103/PhysRevMaterials.3.085201

\section{INTRODUCTION}

The scattering of light by particles is one of the classical problems in the theory of electromagnetism and optics (see classical textbooks [1-3] and the recent review [4]). Initially, the scattering theory (known as Mie scattering theory) was developed for homogeneous spherical particles, for which exact analytical solutions can be derived in the form of multipole expansions. The solutions are obtained as an infinite series of partial spherical waves, called multipoles, where the contribution of each multipole to scattering is given by a numerical weight factor, called the multipole moment. Each multipole moment is associated with a specific charge-current distribution. Furthermore, this exact solution has been extensively studied and successfully applied to describe scattering by particles of various shapes and compositions (see the comprehensive overviews of available theories in Refs. [5-7] and references therein). Besides, considerable effort has been devoted to solving scattering problems with simpler and more compact mathematical formulations targeted for various practical applications [8-12].

From the standpoint of practical applications, it is often desirable to suppress scattering from particles in a given direction. In particular, the suppression of forward and backward scattering are of special interest. Typically, scattering suppression is achieved by realizing multilayered designs of particles $[13,14]$ or covering them with special resistive (thin film) coatings. Such coatings can be made of lossy dielectrics [15] or metals [16,17] depending on the wavelength of the scattered radiation. Recently, graphene has also been proposed as a coating material [18-21]. While this approach is very effective, its implementation at the nanoscale remains a complex task with the current technological resources.

Fortunately, for uncoated particles, the scattering suppression can be also obtained. It appears naturally in magnetic particles, when particular conditions between the permittivity $\varepsilon$ and permeability $\mu$ of the particles are satisfied [22,23]. For a small (subwavelength) magnetic sphere, when the condition $\mu=\varepsilon$ holds, the backward scattering caused by the sphere appears eliminated, whereas the forward scattering is suppressed when the condition $\mu=-\varepsilon$ holds. This scattering suppression is known as the Kerker effect, and from the viewpoint of Mie scattering theory it arises from the resonant overlapping electric-dipole (ED) and magnetic-dipole (MD) moments with the same magnitude (in particular, there is a near-zero backward scattering when the ED and MD moments are in phase, and a strong backward scattering when they are out of phase).

Although these conditions were obtained for a small magnetic sphere, similar scattering characteristics can be achieved for nonmagnetic particles with large enough values of permittivity. For such particles the MD moment also makes a contribution to the scattering in the small-particle limit $[1,22]$, and thus it can overlap the ED moment with the same 
magnitude, when the sphere radius is consciously adjusted. Moreover, there is a possibility of similar overlapping for other higher-order moments [e.g., electric quadrupole (EQ) and magnetic quadrupole (MQ) moments and so on]. The manifestation of interplay between the multipolar moments in the scattering by particles of an arbitrary shape under different radiation conditions is now referred to as the generalized Kerker effect [24,25] (see also extensions specific for the generalized Kerker effect listed in Ref. [26]).

While the mechanism of suppression of forward and backward scattering on a single dielectric particle mediated by the Kerker effect is well described, there is a special interest in the arrangement of such particles into a lattice in order to design functional metasurfaces with desired reflection and transmission characteristics [10]. In particular, one can utilize the generalized Kerker effect for the metasurface composed of dielectric particles to suppress reflection from the high-index substrate, and in this case, the metasurface serves as an antireflective coating $[27,28]$. One important class of such structures are reflectionless all-dielectric Huygens' metasurfaces operating on spectrally overlapped MD and ED moments oriented perpendicular to each other with equal magnitude $[29,30]$.

One significant advantage of all-dielectric metasurfaces is the possibility to fabricate them on a traditional silicon-oninsulator (SOI) platform. Unfortunately, considering permittivity of the bare silicon in the visible part of the spectrum desired for operation (with the typical diameter of particles in the range of 50-200 nm), the Kerker effect conditions cannot be satisfied for spherical particles. Nevertheless, by adjusting the diameter and thickness of cylindrical particles (disks) or the width and height of rectangular particles (cubes), it is possible to bring the corresponding moments into overlap. Moreover, metasurfaces based on silicon particles made in the form of disks or cubes are easier to fabricate at the nanoscale by utilizing modern lithography technologies.

Although the technologies of manufacturing of silicon nanoparticles are well established today, production of alldielectric metasurfaces at the nanoscale still requires expensive equipment and materials [31]. It can be also time consuming. In this regard, correct modeling and prototyping of nanostructures become very important. Accordingly, in order to confirm developed concepts of all-dielectric nanostructures, quasi-optic (microwave) experiments can be used. For the microwave range, it is often much easier and cheaper to fabricate the required samples with micron resolution by using modern milling machines, laser cutting, and three-dimensional (3D) printing technologies. Moreover, the sources of the microwave radiation are also relatively inexpensive.

In the present paper, we study and implement metasurfaces which allow the suppression of the reflected fields. In contrast to Huygens' metasurfaces, the transmitted light traverses the metasurface without perturbation of its amplitude and phase, therefore displaying lattice perfect transmission accompanied with strong nontrivial near fields. The constitutive building blocks (i.e., meta-atoms) of the transparent metasurfaces are "transverse scatterers" and have been extensively investigated in our previous work [32]. We show here that the duality of the Kerker and anti-Kerker conditions governing the transverse scattering of single scatterers still holds for hightransparency lattices in the subdiffractive regime regardless of

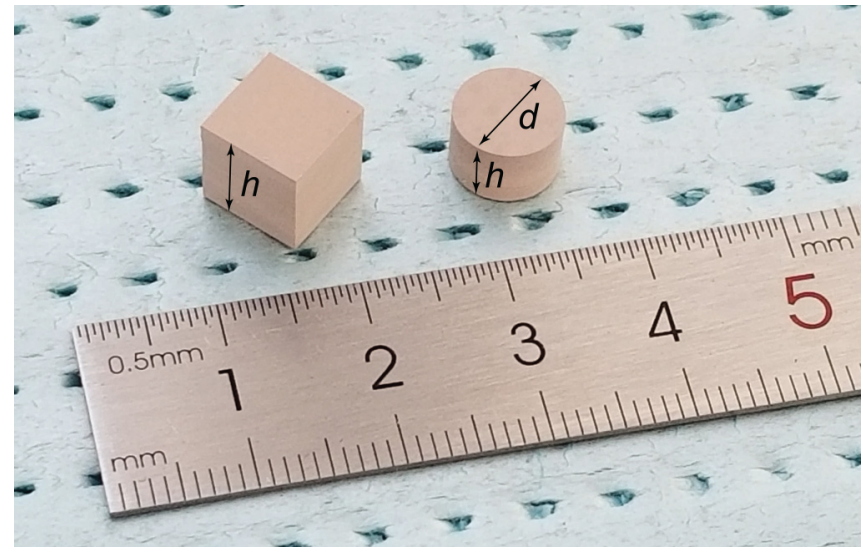

FIG. 1. Dielectric (ceramic) particles under study. The geometrical parameter $h$ represents the cubic particle edge and the disk's height while $d$ gives the disk's diameter.

variations in the lattice parameter. We prove experimentally in the microwave range that both the amplitude and phase of the transmitted wave remain unaffected. The multipole decomposition semi-analytic approach of a single particle scattering has been extended to decompose the reflection and transmission coefficients of a subdiffractive lattice system in a homogeneous medium with or without the presence of a substrate.

By exposing the coupling extent between multipole resonances, we find that the zero-reflection point is not affected by variations in the lattice spacing within the subdiffractive region. Finally, we present a different mechanism of reflection suppression from a dielectric metasurface placed near a conducting sheet. In this case, we show that the light is fully absorbed at the magnetic quadrupole resonance by exploring the link between the resonant properties of the stand-alone dielectric cavity and the optical response of the metasurface.

\section{FAR-FIELD PROPERTIES OF ALL-DIELECTRIC METASURFACES}

Our metasurface configuration consists of periodically arranged dielectric particles in an infinite lattice in the $x-y$ plane (Fig. 1). We assume that the scattered field of each particle is fully characterized by the induced multipole moments up to quadrupoles. Thus, the total electric field outside the lattice is the summation of the scattering from all multipoles over the lattice geometry,

$$
\mathbf{E}(\mathbf{r}) \cong \mathbf{E}_{\text {inc }}(\mathbf{r})+\sum_{\mathbf{r}_{\|}}^{\infty}\left[\hat{g}^{E p} \cdot \mathbf{p}+\hat{g}^{E m} \cdot \mathbf{m}+\hat{g}^{E Q}: \hat{Q}+\hat{g}^{E M}: \hat{M}\right],
$$

characterized by the Green tensors [33]

$$
\begin{aligned}
g_{i j}^{E p} & =\frac{1}{\varepsilon_{0}} \partial_{i j} g_{0}\left(\mathbf{r}-\mathbf{r}_{\|}\right), \\
g_{i j}^{E m} & =\frac{i k}{c \varepsilon_{0}} \epsilon_{i s j} \partial_{s} g_{0}\left(\mathbf{r}-\mathbf{r}_{\|}\right), \\
g_{i j l}^{E Q} & =-\frac{1}{\varepsilon_{0}}\left(\partial_{l} \partial_{i j}+\partial_{j} \partial_{i l}\right) g_{0}\left(\mathbf{r}-\mathbf{r}_{\|}\right), \\
g_{i j l}^{E M} & =-\frac{i k}{c \varepsilon_{0}}\left(\epsilon_{i j m} \partial_{m l}+\epsilon_{i l m} \partial_{m j}\right) g_{0}\left(\mathbf{r}-\mathbf{r}_{\|}\right),
\end{aligned}
$$


where $g_{0}(\mathbf{r})$ is the scalar Green's function, $\mathbf{r}$ denotes a point in space relative to the coordinate origin, $\mathbf{E}_{\mathrm{inc}}$ is the incident field, $\epsilon_{i s j}$ is the Levi-Civita symbol, and $\partial_{i j}=\partial_{i} \partial_{j}-k^{2} \delta_{i j}$. The vector $\mathbf{r}_{\|}=n \mathbf{a}_{x}+m \mathbf{a}_{y}$ represents the positions of the particles, where $\mathbf{a}_{x}, \mathbf{a}_{y}$ are lattice vectors and $n, m$ are integers. The term $\hat{g}^{E p} \cdot \mathbf{p}$ gives the sum of the field scattered by the EDs. Similarly, $\hat{g}^{E m} \cdot \mathbf{m}, \hat{g}^{E Q}: \hat{Q}$, and $\hat{g}^{E M}: \hat{M}$ represent the contribution of the MD, EQ, and the MQ to the total field, respectively. The summation in Eq. (1) includes evanescent and propagating waves. The latter correspond to open diffraction channels contributing to the far field. In the subdiffractive region, the reflection and transmission of the lattice are characterized by the first-order diffraction, providing nonzero contributions to the far-field [34,35]. Knowledge of the field outside the lattice allows the formulation of the non-Fresnel reflection $z>0$ and transmission $z<0$ coefficients of the lattice as

$$
r_{m}=\frac{\left(\mathbf{E}-\mathbf{E}_{\mathrm{inc}}\right) \cdot \mathbf{E}_{\mathrm{inc}}^{*}}{\mathbf{E}_{\mathrm{inc}} \cdot \mathbf{E}_{\mathrm{inc}}^{*}}, \quad t_{m}=\frac{\mathbf{E} \cdot \mathbf{E}_{\mathrm{inc}}^{*}}{\mathbf{E}_{\mathrm{inc}} \cdot \mathbf{E}_{\mathrm{inc}}^{*}} .
$$

These coefficients are complex numbers even for lossless dielectric particles and contain the characteristics of the lattice periodicity. Throughout this work, we consider that the metasurfaces under study are illuminated by a normally incident, linearly polarized plane wave $\mathbf{E}_{\text {inc }}=E_{0} e^{i k z} \mathbf{x}$, where $\mathbf{x}$ is a unit vector directed along the $x$ axis. Equation (3) can then be reduced to [10]

$$
\begin{aligned}
r_{m} & \cong \frac{i k}{2 E_{0} A \varepsilon_{0}}\left(p_{x}+\frac{1}{c} m_{y}-\frac{i k}{6} Q_{x z}-\frac{i k}{2 c} M_{y z}\right), \\
t_{m} & \cong 1+\frac{i k}{2 E_{0} A \varepsilon_{0}}\left(p_{x}-\frac{1}{c} m_{y}+\frac{i k}{6} Q_{x z}-\frac{i k}{2 c} M_{y z}\right),
\end{aligned}
$$

where $c$ is the speed of light in vacuum and $A=\left|\mathbf{a}_{x} \times \mathbf{a}_{y}\right|$ is the area of the unit cell. The last equations link the scattering pattern of a single particle and the far-field wavefront of the lattice, thus allowing us to study the characteristics of the field emitted by the lattice by means of the multipole decomposition of the field scattered by a single meta-atom of the lattice. We previously found the conditions at which the forward and backward scattering of an isolated scatterer are nearly suppressed simultaneously [32]. Similar conditions can be obtained for a fully transparent lattice in a homogeneous medium directly by setting the lattice reflection and its contribution to transmission to zero, $r_{m}=0$ and $1-t_{m}=0$, resulting in

$$
p_{x}=\frac{m_{y}}{c}, \quad \frac{Q_{x z}}{3}=\frac{M_{y z}}{c}, \quad p_{x}=-\frac{k M_{y z}}{2 i c} .
$$

Previously, we assumed that the scattering is fully characterized by the multipole contributions up to the MQ; however, these conditions can be further extended to include higherorder multipoles. The first term of Eq. (5) is the well-known Kerker condition for dipoles and the second term is the Kerker-like condition for quadrupoles. The last term is of particular interest since it suggests that the coherent dipoles are in a $\pi$ phase relation with respect to the coherent quadrupoles or the anti-Kerker condition of the dipole-quadrupolar scatterers. Simultaneous fulfillment of the Kerker and anti-Kerker conditions leads to the redirection of the scattered power to the lateral directions and enhanced suppression of the contributions to reflection and transmission [32], leaving the incident wave almost unperturbed.

We have further validated our analytical predictions against numerical simulations and experiments. In accordance with our available experimental means, we have chosen the microwave range of the spectrum $(8-15 \mathrm{GHz})$ to demonstrate the manifestation of the transparency effect. We consider particle arrangements constituted of a low-loss, high-permittivity microwave ceramic, manufactured in the form of disks and cubes (Fig. 1).

For metasurfaces constituted of such particles, we performed numerical simulations in COMSOL Multiphysics ${ }^{\circledR}$. The electromagnetic response of the metasurfaces was obtained by imposing Floquet-periodic boundary conditions on four sides of the unit cell to simulate an infinite two-dimensional (2D) array of particles. Figure 2 shows the amplitudes and phases of the simulated reflection and transmission coefficients for two particular metasurfaces constituted of disks [Fig. 2(a)] and cubes [Fig. 2(b)] organized in a square lattice with spacing $a=\left|\mathbf{a}_{x}\right|=\left|\mathbf{a}_{y}\right|$. The simulations confirm that in the chosen frequency band areas of complete transparency indeed exist. We mark corresponding resonant frequencies by vertical dashed lines (they are $f_{\mathrm{sp}}=10.5 \mathrm{GHz}$ and $f_{\mathrm{sp}}=9.67 \mathrm{GHz}$ for the array of disks and the array of cubes, respectively).

We decompose the reflection spectra in Fig. 2. As a first remark, we note that the near-zero-reflection point is located in the vicinity of the Fano-like profile of the ED resonance. Second, the MQ resonance appears on the red side of the near-zero-reflection point for the array of disks and on the blue side for the cubic array, while the other multipoles are nonresonant. Consequently, conditions (5) are nearly satisfied; the four leading multipoles have comparable amplitudes at the zero-reflection point. The dipoles are in-phase (Kerker) and the same situation takes place for quadrupoles (generalized Kerker). The last term of conditions (5), as depicted by the black dashed line, shows the coherent dipoles are in antiphase with the coherent quadrupoles. In contrast to the well-known Huygens' metasurfaces where strong forward scattering results in a phase difference between the incident and transmitted fields; here we notice both waves are in phase (blue lines), meaning this kind of metasurfaces are extraordinarily transparent with almost no change of amplitude or phase of the transmitted wave.

We should note that a similar scenario of tuning dipolequadruple resonances is also reported in Ref. [36] for an onion-like three-layer folded metal metasurface with dielectric separations. However, the rod structure enhances scattering in the forward direction with several orders of magnitudes which resembles the generalized Kerker effect of an electromagnetic dipole-quadrupolar scatterer.

\section{EFFECT OF LATTICE SPACING, MATERIAL LOSSES, AND INCIDENCE}

Variations in the lattice spacing influence the optical response of a metasurface in an equivalent way to changes in the shape and composition of the meta-atom. The spectral position of the electric and magnetic modes in a lattice can be tailored by adjusting spacing in one of the lattice axes while 
(a)

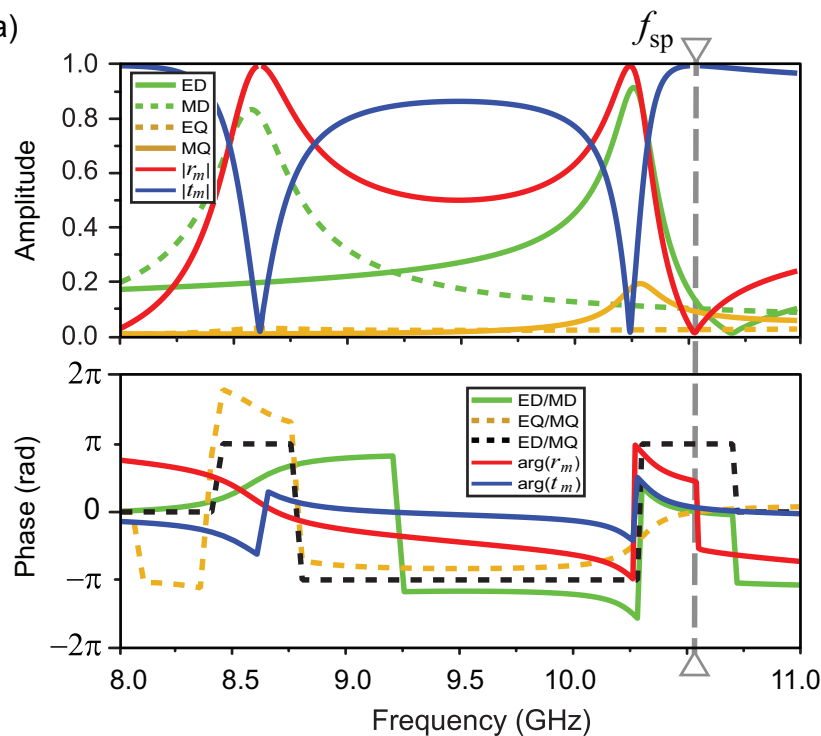

(b)

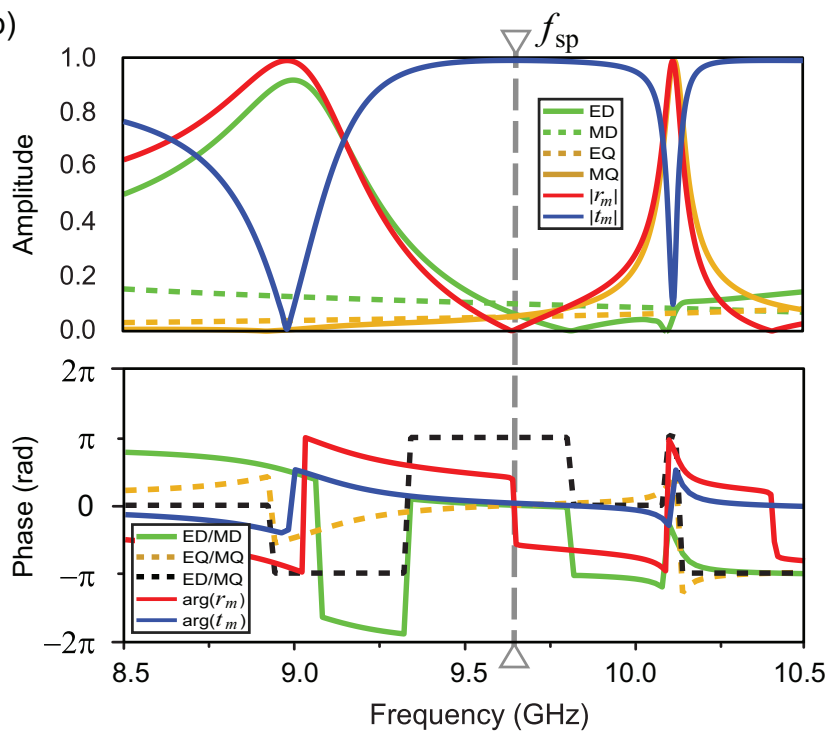

FIG. 2. Simulated reflection $\left(r_{m}\right)$ and transmission $\left(t_{m}\right)$ coefficients, and multipole decomposition of an all-dielectric lossless metasurface composed of (a) disk and (b) cubic particles. The decomposition is done by using Eq. (4) and the vertical lines indicate the spectral position of the transverse Kerker effect (transparency effect). The geometrical and material parameters of the metasurfaces under study and their constituting building blocks are (a) $d=$ $8.0 \mathrm{~mm}, h=5.0 \mathrm{~mm}, a=20 \mathrm{~mm}, \varepsilon=23$; (b) $h=8.0 \mathrm{~mm}, a=$ $22 \mathrm{~mm}, \varepsilon=21$.

keeping the other constant, in such a fashion that one can bring both resonances to overlap and suppress reflection [30,37]. We show here that, although the Fano profile of the ED and MQ resonances are spectrally shifted in different directions, the frequency of the suppressed reflection corresponding to their shared dip point is fixed on the spectrum. We restrict ourselves to the consideration of the array of cubes; however, the discussion could be applied to all similar scenarios that satisfy the conditions (5).

We impose the constraints that the lattice is axially symmetric for normally incident light. The effective field acting on each particle center is the summation of the incident field and all of the multipole radiations in the lattice. Furthermore, from the definition of the Green tensors that describe multipole scattering [Eq. (2)], the coupling between the ED and both MD and EQ are canceled out due to the lattice symmetry. The $\mathrm{MQ}$, on the other hand, is very small in the region where the ED Fano resonance appears, so we count only for the ED-ED coupling, which can be formulated over the lattice geometry as

$$
\hat{d}=\sum_{n \neq 0} \hat{g}^{E p}\left(n \mathbf{a}_{x}, 0,0\right)+\sum_{n} \sum_{m \neq 0} \hat{g}^{E p}\left(n \mathbf{a}_{x}, m \mathbf{a}_{y}, 0\right),
$$

where $\hat{d}$ is a second-order tensor representing the dipoledipole coupling. Here the summation is split over the lattice geometry to a summation over one of the coordinate axis (the first part) and a summation over all the remaining lines in the lattice. The excitation wave setup restricts the excited electric multipole components to the plane of incidence [9]. In the case of an $x$-polarized incident field, only the $p_{x}$ component of the ED is induced. Also, due to the lattice symmetry, all components of the periodic tensor $\hat{d}$ except the diagonals are zero. We write $[33,38-40]$

$$
p_{x} \cong \frac{\alpha_{p} E_{0}}{1-\alpha_{p} d_{x x}},
$$

with

$$
\begin{aligned}
d_{x x} \cong & -\frac{2 k^{2}}{a}\{\ln [2-2 \cos (k a)]+i \pi-k a\} \\
& +\sum_{n \neq 0} \frac{2 i n k a-1}{n^{3} a^{3}} e^{i n k a}+\sum_{n} \sum_{m \neq 0} \frac{\omega_{n}^{2}}{2 a^{2}} K_{0}\left(-i m a \omega_{n}\right),
\end{aligned}
$$

where $\alpha_{p}$ is the ED polarizability of the individual particle, $K_{0}$ is the Bessel function of the second kind, and $\omega_{n}=$ $\left[k^{2}-(2 \pi n / a)^{2}\right]^{1 / 2}$. The lattice effect (coupling) is determined by the spacing and rises rapidly closer to and around the first diffraction order $[39,40]$. This spectral position where the wavelength equals the lattice spacing $\lambda=a$ is referred to as the first diffraction order or Rayleigh anomaly. The energy confinement in the first diffraction order causes the coupling to diverge instantaneously. Upon inspection of Eq. (7), one can see that the effective multipole moments change when the product $\left(\alpha_{p} d_{x x}\right)$ in the denominator becomes significant. Hence both the ED polarizability and the coupling are equally relevant and need to be considered in order to determine the limitations of the fixed-position reflection.

Figure 3 shows the reflection and transmission spectra for different lattice spacing, where we have considered the array of cubes with and without accounting for material losses. In the subdiffractive region, the low reflection point at the transverse scattering $\left(f_{\mathrm{sp}}=9.67 \mathrm{GHz}, \lambda_{\mathrm{sp}}=31 \mathrm{~mm}\right)$ is fixed while other low reflection points are shifted. Also there are opposite spectral displacements of the ED and MQ modes leading to consequent broadening or narrowing of the lowreflection region. It was previously reported [39] that the evolution of ED-ED coupling along one-dimensional (1D) arrays results in an increase of the interaction between dipoles with a wider spacing, while the diffraction orders work as a 


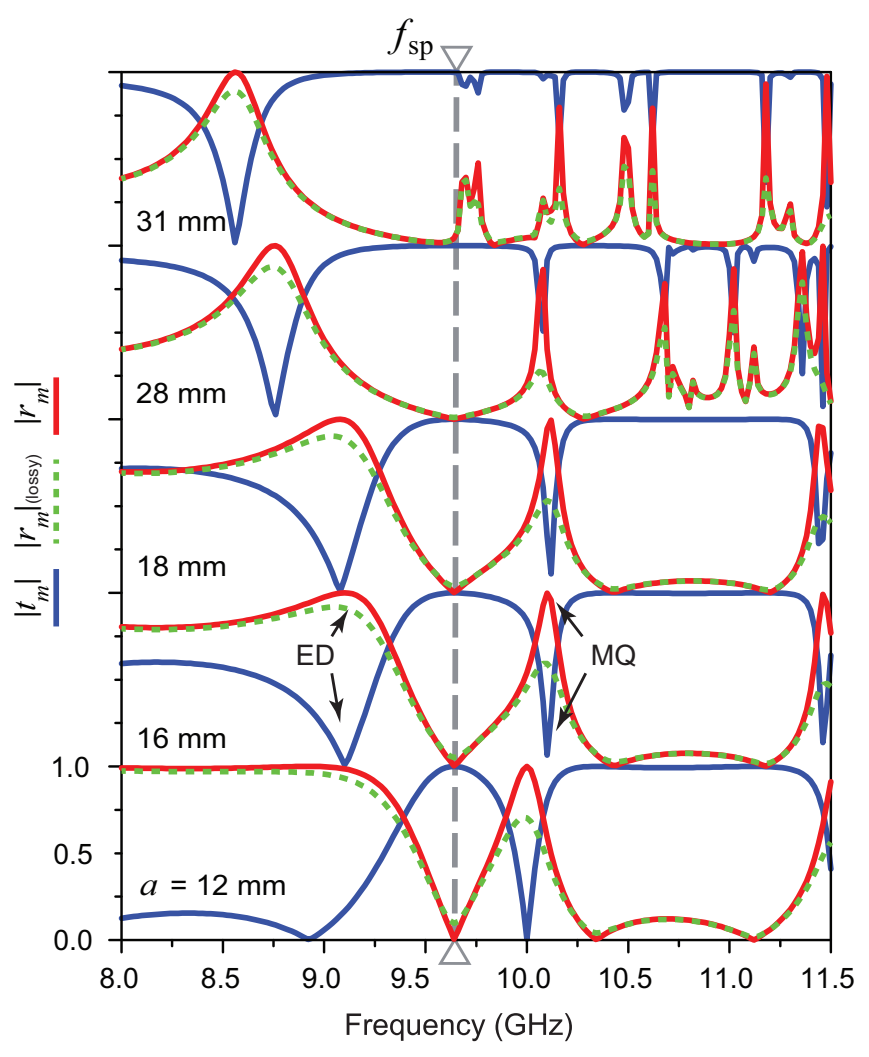

FIG. 3. Calculated reflection and transmission coefficient spectra for different lattice spacing of a square lattice constituted of our ceramic cubes. Dashed green curves show the reflection from the metasurface where the real material losses have been taken into account $\left(\tan \delta=8 \times 10^{-3}\right)$. The vertical gray dashed line indicates the fixed spectral position of the reflection suppression point between the electric-dipole (ED) and the magnetic-quadrupole (MQ) resonances (protected-like transparency point). The geometrical parameters of the particles are the same as in Fig. 2.

divergence and reset the points. The same goes for 2D lattices [see the last term in Eq. (8)], since it can be considered as an infinite summation of 1D lattices [41]. We notice here that the Fano profile of ED is deformed and weakened as the ED-ED coupling increases. The spectral regions with a resonant particle polarizability enable a dramatic change in the reflection behavior since the term $\left(\alpha_{p} d_{x x}\right)$ becomes significant and influences the effective dipole moment. Therefore, as can be seen in Fig. 3, the speed of shifting is not homogeneous. Nevertheless, since the zero-reflection point has a very low polarizability and, therefore, is fixed within the subdiffractive range of spacing. Note that, if spacing is very small compared with the wavelength $a \ll \lambda$, the higher-order multipoles are excited and the aforementioned discussion is no longer applicable.

Now let us examine the lattice response beyond the first diffraction order $(\lambda=31 \mathrm{~mm})$. In this case there is a divergent coupling along with the Fano profile dip. If the spacing is beyond this limit, a new lattice-forced ED Fano profile leads to revoking the zero-reflection point [40]. Contrary to the ED, the MQ resonance shifts to the blue side of the spectrum until it becomes deteriorated by higher diffraction orders when the lattice spacing is increased (see Fig. 3). However, according to the characteristics of the periodic Green tensors, the EDMQ (MD-EQ) coupling is nonvanishing due to the lattice symmetry [33]. For instance, the coupling between MD and EQ in a symmetric lattice of finite size in a homogeneous medium causes a shift in the EQ resonance [42]. In the current profile, the ED contribution to the reflection and transmission at the frequency of the MQ resonance is sufficient (Fig. 2) and their coupling causes a blueshift in the MQ resonance. One can notice close to the first diffraction order (see curves for the lattice spacing $a=28$ and $31 \mathrm{~mm}$ ) on the short-wavelength side that the strong coupling between the modes of the lattice causes a series of reflection and transmission resonances and eventually dominates the ED-MQ subdiffractive coupling. In general, the opposite displacements of the ED and MQ resonances lead to a broadening of the low-reflection area around the zero-reflection point.

We now proceed to analyze the effect of the particle inherent losses on the reflection suppression. From Fig. 3 (see the dashed green line), we find the reflection to become slightly enhanced at the point of interest; however, no change in its spectral position can be appreciated. In fact, losses decrease the $Q$ factor of the Fano resonance. Resonances in subwavelength open dielectric resonators are governed by the imaginary part of the permittivity $[43,44]$ because $Q \backsim$ $\operatorname{Re}(\varepsilon) / \operatorname{Im}(2 \varepsilon)$. With the current permittivity of ceramic particles $\varepsilon=21+0.168 i$ and $Q \backsim 63$, we can conclude that, for example, if our lattice was constituted of silicon particles in the near-infrared region, where the quality factor can exceed several thousand, our spectra would display an almost-zeroreflection point.

For the sake of the completeness of our investigation of the protected-like transparency point, we plot several cases of oblique angle incidence $(\varphi \neq 0)$ in Fig. 4 for both $x$ and $y$ polarizations of the incident wave. For oblique incidence, the first diffraction order is effectively moved to shorter wavelengths [45] and the particles receive nonuniform incident field depending on the angle on incidence. However, with a small angle deviation from the normal incidence (bounded to $15^{\circ}$ ), our system shows very low reflection at the wavelength of the transverse scattering. Moreover, the protected-like transparency point is more stable for the $y$-polarized wave, surviving even for large angles of incidence (Fig. 4).

\section{EXPERIMENTAL DEMONSTRATIONS}

To verify the theoretical and numerical predictions, we manufactured and tested two square metasurfaces, each one containing $18 \times 18$ unit cells and having the side length of $400 \mathrm{~mm}$. Their unit cells correspond to those presented in Figs. 2(a) and 2(b) for the metasurfaces composed from disks and cubes, respectively. As a dielectric material, we used the Taizhou Wangling TP-series microwave ceramic characterized by the relative permittivity $\varepsilon=23$ for disks and $\varepsilon=21$ for cubes. The loss tangent for the ceramic is $\tan \delta \approx 6 \times 10^{-3}$ at $10 \mathrm{GHz}$. The dielectric particles with the sizes mentioned in the caption of Fig. 2 were fabricated with the use of precise mechanical cutting techniques. To arrange them into a lattice, an array of holes was milled in a custom holder made of a styrofoam material whose permittivity is $\varepsilon_{s}=1.05$ and thickness of the plate is $h_{s}=20.0 \mathrm{~mm}$. Furthermore, its 


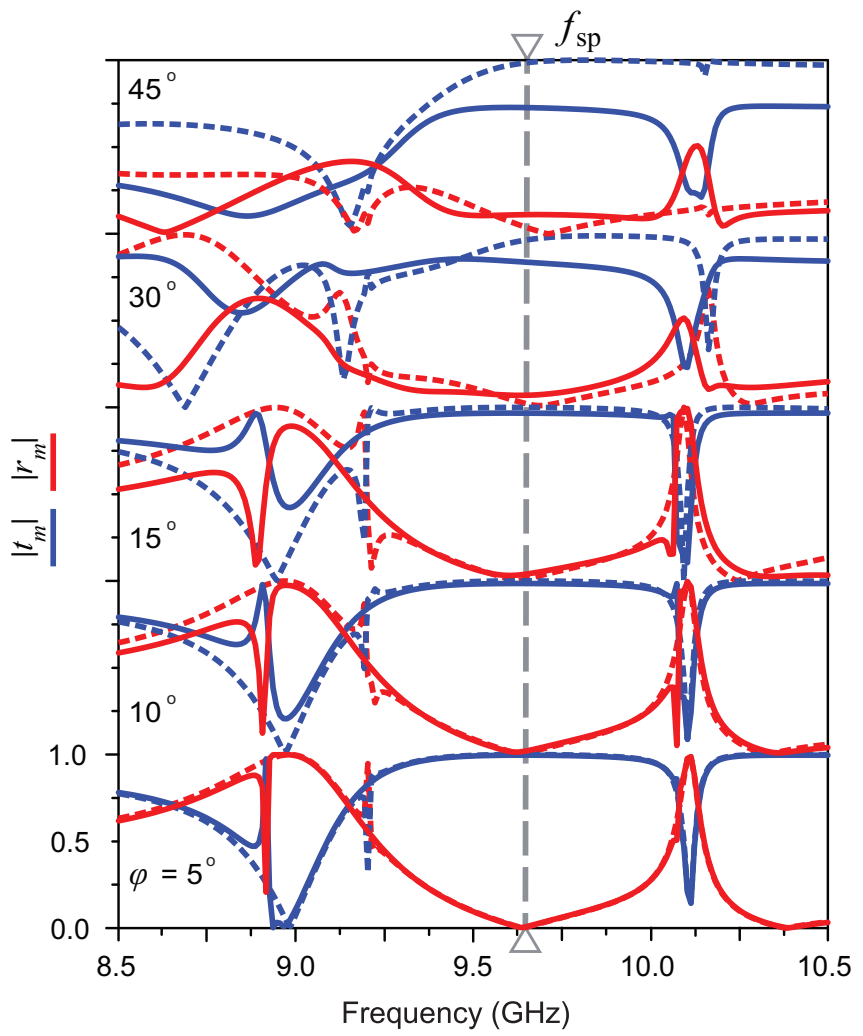

FIG. 4. Calculated reflection and transmission coefficient spectra for different angles of incidence of the illuminating wave for a metasurface constituted of ceramic cubes. Solid and dashed lines are related to the $x$ - and $y$-polarized waves, respectively. The vertical gray dashed line indicates the protected-like transparency point for the normally incident wave. The geometrical parameters of the particles are the same as in Fig. 2.

complex reflection and transmission coefficients were measured in the same frequency range as in the simulations by using the common technique where the measurements are performed in the radiating near-field region and then transformed to the far-field zone.

The measurement procedure as well as experimental setup are described in detail in Refs. [46,47]. During the investigation, the prototype was fixed at $2.0 \mathrm{~m}$ from a rectangular linearly polarized broadband horn antenna. The antenna generated a quasi-plane-wave with the required polarization. The antenna was connected to the first port of the Keysight E5071C Vector Network Analyzer (VNA) by a $50 \mathrm{Ohm}$ coaxial cable. To detect the electric field, an electrically small dipole probe connected to the second port of the VNA was used.

The measured transmission and reflection coefficients for both metasurfaces are collated in Fig. 5. For comparison, additional numerical simulations were performed, taking into account the measured deviations in metasurface dimensions from the nominal values appeared in fabrication as well as losses in the particles. The data presented demonstrate very good agreement between measurements and simulations, where one can see the areas discussed above of complete transparency.
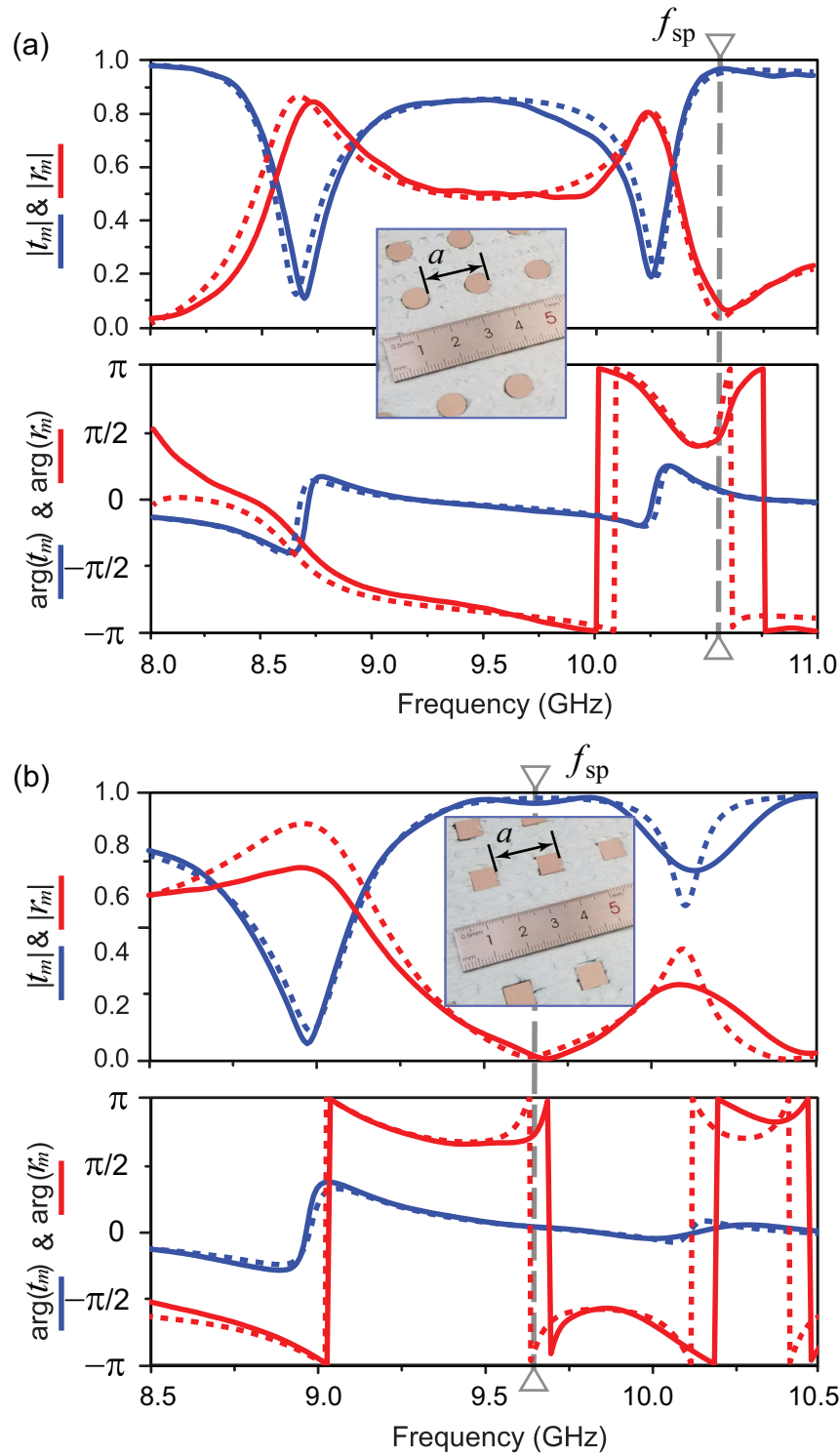

FIG. 5. Simulated (dashed lines) and measured (solid lines) transmission $\left(t_{m}\right)$ and reflection $\left(r_{m}\right)$ coefficients of the all-dielectric metasurface composed of (a) disk and (b) cubic particles. The insets demonstrate fragments of the metasurface prototypes. In the simulation the substrate is modeled as a lossless dielectric with near-unity refractive index, while actual material losses in ceramic particles are taken into account, where (a) $\varepsilon=23+i 0.138$, (b) $\varepsilon=21+i 0.168$, and all geometrical parameters of the particles and lattice spacing are the same as in Fig. 2.

\section{METASURFACES PLACED ON A CONDUCTING SUBSTRATE}

For various practical realizations, where the lattice is to be placed on a substrate, we extend the semi-analytic derivation of the reflection and transmission decomposition of the lattice shown in Sec. II to account for a substrate. We found the transverse scatterers gradually transform to Kerker scatterers on high-index substrates [32], and the broadband Huygens' metasurfaces can be realized as a result of this mechanism [29]. Nonetheless, in this section we discuss the transformation of the considered metasurface to a fully absorbing lattice 


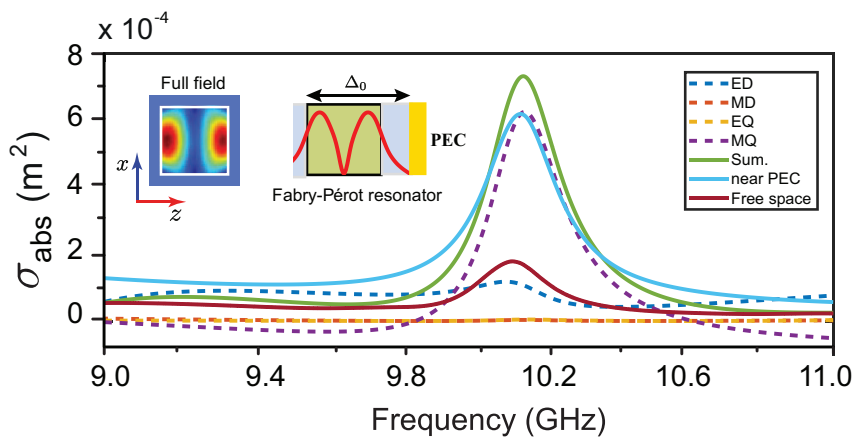

FIG. 6. Absorption cross section and its approximated multipole decomposition of the stand-alone ceramic cube in the two situations presented in the text, i.e., free space and placed $3 \mathrm{~mm}$ above the PEC sheet. The left inset shows the calculated electric-field distribution associated with the MQ at resonance for the cube above the PEC sheet (the point of maximal absorption), and the right inset depicts the electric-field amplitude of the $m=5 / 2$ eigenmode for the equivalent 1D Fabry-Pérot resonator. The material and geometrical parameters of the cube are the same as in the metasurface in Fig. 5(b).

with the addition of a conducting substrate (a PEC sheet in the microwave range). Analytic results will be supported by numerical calculations and experimental measurements.

We start our discussion with an investigation of the spectral behavior of an isolated meta-atom, i.e., a single cube (introduced in Fig. 1) deposited over a PEC sheet. Since the holder has a finite thickness, there can be a distance (gap) between the particles and the PEC sheet. We suppose that the gap is much smaller than the wavelength of the wave in the substrate material (in our experimental samples the gap is $3 \mathrm{~mm}$ ). Hereinafter we account for this gap between the cubes and the sheet.

To provide better physical intuition, let us follow Ref. [48] and consider only spatial variations along the height of the cavity. In the 1D case, an open cavity can be seen as a lossy dielectric Fabry-Pérot resonator of refractive index $n=\sqrt{\varepsilon}$. After excitation with an external pulse (the incident wave), the resonator supports a series of standing-wave-like field patterns whose excitation depends on the number of effective wavelengths that can be introduced inside the cavity of length $\Delta_{0}$ (see inset of Fig. 6). In the general case, the walls of the resonator have nonzero transmittance and thus allow the confined modes to leak into the environment in the form of outgoing plane waves. When the internal field interferes constructively with itself, mode resonances occur. The latter take place only when the phase shift induced in the circulating wave after reaching the second wall and returning to the first accounts for $2 \pi$. Using this fact, it is straightforward to show that the resonant frequencies for a lossless dielectric Fabry-Pérot resonator are [49]

$$
f_{m}=\frac{c m}{2 n \Delta_{0}},
$$

where $m$ is an integer or half-integer number accounting for the different resonance modes [50]. If losses are considered, an imaginary term needs to be added to the expression for $f_{m}$ [48], but the real part remains the same as in Eq. (9). Higher values of $m$ allow us to contain additional quarter wave- lengths inside the resonator. For example, for the first three $m=1 / 2,1,3 / 2$, a quarter wavelength, half wavelength, and three quarters of a wavelength can be fit in the length $\Delta_{0}$, respectively. In the general case, the effective wavelengths that can be fit in the resonator for a given $m$ are $\Delta_{0} / \lambda_{\text {eff }}=m / 2$, where $\lambda_{\text {eff }}=\lambda / n$ and $\lambda$ is the wavelength in free space.

In Fig. 6 we show the numerically calculated absorption cross section of the cubic particle above PEC substrate, its multipole decomposition, and insets showing the link between the aforementioned Fabry-Pérot mode and the resonant MQ.

The individual contribution of the leading multipoles was calculated approximately as the difference between the extinction and scattering cross sections $[9,11]$. First, we considered the exact expressions for the extinction cross sections of the electric and magnetic dipoles and quadrupoles [9,51]:

$$
\begin{aligned}
\sigma_{\mathrm{ext}}^{p} & =\frac{\omega}{2 I_{0}} \operatorname{Im}\left\{\mathbf{p} \cdot \mathbf{E}_{\mathrm{exc}}^{*}\left(\mathbf{r}_{0}\right)\right\}, \\
\sigma_{\mathrm{ext}}^{m} & =\frac{\omega \mu_{0}}{2 I_{0}} \operatorname{Im}\left\{\mathbf{m} \cdot \mathbf{H}_{\mathrm{exc}}^{*}\left(\mathbf{r}_{0}\right)\right\}, \\
\sigma_{\mathrm{ext}}^{Q} & =\frac{\omega}{12 I_{0}} \operatorname{Re}\left\{i \hat{Q}:\left.\nabla \mathbf{E}_{\mathrm{exc}}^{*}\right|_{\mathbf{r}=\mathbf{r}_{0}}\right\}, \\
\sigma_{\mathrm{ext}}^{M} & =-\frac{1}{2 I_{0}} \operatorname{Re}\left\{\left.[\nabla \times(\hat{M} \cdot \nabla)] \cdot \mathbf{E}_{\mathrm{exc}}^{*}\right|_{\mathbf{r}=\mathbf{r}_{0}}\right\},
\end{aligned}
$$

where $\omega$ is the angular frequency, $I_{0}$ is the incident energy flux, and $\mathbf{E}_{\mathrm{exc}}^{*}\left(\mathbf{r}_{0}\right)$ and $\mathbf{H}_{\mathrm{exc}}^{*}\left(\mathbf{r}_{0}\right)$ are the complex conjugates of the excitation electric and magnetic fields evaluated at the center of the particle $\mathbf{r}_{0}$. The expressions above are valid for any excitation field and particles of arbitrary shape. For an $x$-polarized plane wave normally incident on a PEC substrate along the $z$ axis, the exciting field which needs to be substituted in Eq. (10) corresponds to the sum of the incident and reflected waves. Second, the individual contributions of each multipole to absorption are assumed to be

$$
\sigma_{\mathrm{abs}}^{L}=\sigma_{\mathrm{ext}}^{L}-\sigma_{\mathrm{sca}}^{L},
$$

where $L$ denotes any of the leading multipole moments. The formulas for each term in the scattering cross-section decomposition are taken from Ref. [11]. Therefore, the approximate total absorption cross section can be written in the form

$$
\sigma_{\mathrm{abs}} \cong \sigma_{\mathrm{abs}}^{p}+\sigma_{\mathrm{abs}}^{m}+\sigma_{\mathrm{abs}}^{Q}+\sigma_{\mathrm{abs}}^{M} .
$$

Here we neglect magnetoelectric coupling between the multipoles due to the PEC [51], and other cross terms between the multipoles. However, Fig. 6 shows that Eq. (12) is in good quantitative agreement with the numerically calculated absorption cross section obtained with COMSOL Multiphysics, indicating the validity of the approach used in the spectral range considered.

Consequently, in Fig. 6 we observe a strong enhancement of the absorption peak centered at the eigenfrequency in comparison with the free-space scenario. Moreover, the induced magnetic quadrupole responsible for this enhancement is fully driven by the eigenmode (standing wave) of the Fabry-Pérot resonator. This is confirmed by the identical internal field distributions of the mode and the one obtained from numerical simulations (insets of Fig. 5). Thus, simple physics of 1D open resonators suffices to qualitatively describe the resonant 
behavior of the single nanoparticle on PEC, as well as the metasurface, as will be discussed shortly.

With the physical intuition gained by the investigation of the single meta-atom, we now turn our attention toward the case of the metasurface. Following the analytical treatment from Sec. II, modeling the metasurface as a plane crossing the center of the particles with non-Fresnel reflection and transmission coefficients (4), and the method given in Refs. [52-54], we can apply the well-known Airy-Fresnel formulas to study the reflection from the whole system. It is similar to the 1D Fabry-Pérot model but is suitable for metasurfaces since it takes into account the mutual particle interaction and provides useful information on each multipole contribution.

Now, $\Delta_{h}$ is the distance from the substrate interface to the plane, where point-like multipoles are localized (particle centers), considered as an imaginary interface [52-54]. These two boundaries now play the role of the walls of a resonator. The reflection and transmission coefficients of the whole system take the form

$$
r_{\mathrm{tot}}=r_{m}+\frac{r_{s} t_{m}^{2} e^{2 i k \Delta_{h}}}{1-r_{s} r_{m} e^{2 i k \Delta_{h}}}
$$

and

$$
t_{\mathrm{tot}}=\frac{t_{s} t_{m}}{1-r_{s} r_{m} e^{2 i k \Delta_{h}}},
$$

where $r_{\text {tot }}$ and $t_{\text {tot }}$ are the overall system reflection and transmission with the substrate, and $r_{s}$ and $t_{s}$ are Fresnel coefficients of the substrate for the normally incident wave. When a PEC is used as substrate (i.e., the reflection of the lower wall of our Fabry-Pérot resonator is -1), the total reflection is simplified to

$$
r_{\text {tot }}=r_{m}-\frac{t_{m}^{2} e^{2 i k \Delta_{h}}}{1+r_{m} e^{2 i k \Delta_{h}}},
$$

where $r_{s}=-1$ and $t_{s}=0$ have been substituted. Therefore, to cancel the reflection and absorb all incident light, the antireflection condition should be fulfilled, and direct reflection from the lattice and the substrate-aided reflection [the second term of Eq. (15)] should be in a $\pi$ phase relation having equal amplitudes [50].

Let us use formula (15) to study the reflection from the metasurface on a substrate. Figure 7 shows the results of the analytical calculations together with the measured reflection and the results of simulations in COMSOL Multiphysics. It is noteworthy that the results are very close to each other, validating the suggested approach.

The multipolar decomposition (15) of $r_{m}$ gives us the particular contributions of the multipoles to the reflection. As is clearly seen from Fig. 6(b), the resonant MQ is once again responsible for the reflection dip (absorption peak, because the PEC interface now allows for nonzero transmission). The spectral position of the MQ resonance at $f_{\mathrm{sp}}=10.25 \mathrm{GHz}$ is slightly shifted in comparison with the free-space case shown in Fig. 2, which is at $f_{\mathrm{sp}}=10.17 \mathrm{GHz}$. However, both positions are in the vicinity of the original MQ resonance of the stand-alone cubic particle (see Fig. 6), underlining that the resonant modes of an isolated meta-atom provide valuable insight into the optical response of the metasurface.

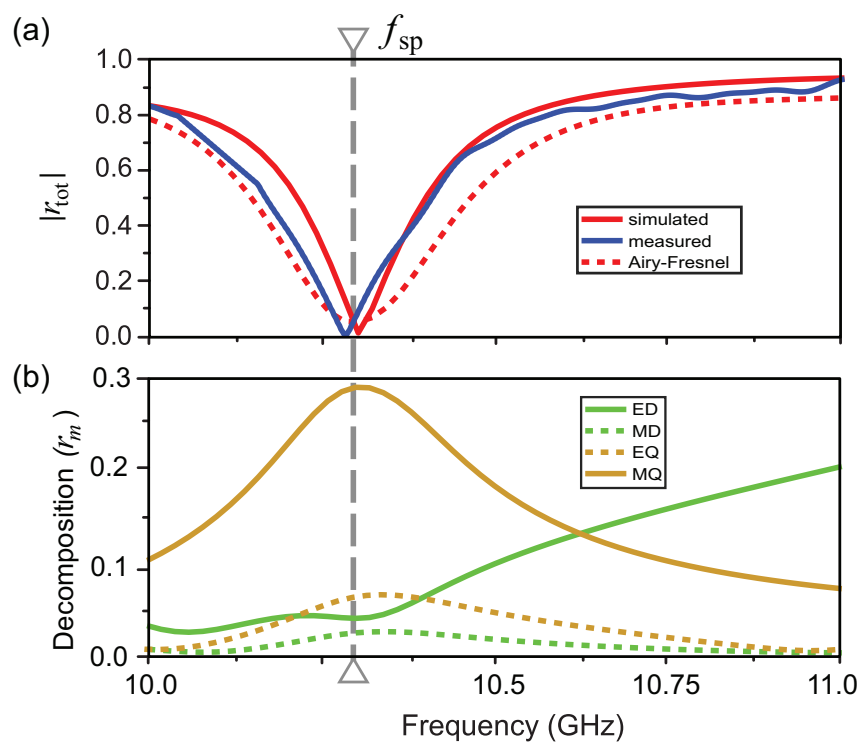

FIG. 7. (a) Simulated and measured total reflection coefficient of the all-dielectric metasurface $\left(r_{\text {tot }}\right)$ composed of cubic particles and placed $3 \mathrm{~mm}$ above the PEC substrate. (b) The multipole decomposition of the first term of Eq. (15) $\left(r_{m}\right)$ which represents the lattice direct contribution to the total reflection. Note here $r_{m}$ has been calculated by taking into account the total excitation field acting on the particles. The material and geometrical parameters of the metasurface are the same as in Fig. 5(b).

\section{CONCLUSIONS}

We have studied, both theoretically and experimentally, a novel class of all-dielectric Mie-resonant metasurfaces governed by the transverse Kerker effect. Such metasurfaces can demonstrate a complete transparency similar to Huygens' metasurfaces, but they experience zero phase shift between the incident and transmitted waves. We have clarified the underlying physics of this effect, and we have formulated the specific conditions for which both reflection and transmission coefficients vanish simultaneously for a square lattice composed of Mie-resonant dielectric particles. This type of optical response occurs when the coherent dipole modes and coherent quadrupole modes excited in the individual dielectric particles satisfy the so-called generalized Kerker condition, and they possess a phase difference between each others. When these conditions are satisfied, the metasurface becomes absolutely transparent, and both amplitude and phase of a transmitted wave are completely unaffected. We demonstrate this effect experimentally for microwave frequencies. Importantly, the coupled multipoles in the lattice do not affect the transparency point, and we have shown that a variation of the lattice spacing in the subdiffractive scattering regime results in either narrowing or broadening of the near-zero-reflection region, and the reflection dip itself does not disappear.

We have studied theoretically and also verified in experiment the effect of the total absorption by the dielectric metasurfaces supporting the transverse Kerker effect placed on a conducting substrate. The qualitative explanation of this effect is based on the Fabry-Pérot resonances, and it shows that the absorption peak is due to a standing wave 
corresponding to the magnetic quadrupole resonance. A more detailed analysis based on the metasurface multipole decomposition and the Airy formulas suggests a good agreement with both numerical simulations and experimental data, and it proves a dominant role played by the MQ resonance in the metasurface absorption.

Thus, the dielectric metasurfaces governed by the transverse Kerker effect can provide both transparency and full absorption, and they could be useful for a design of a variety of optical elements with enhanced properties, allowing for more flexible manipulation of light with ultrathin planar optics. Moreover, strong near-fields mediated by the almost-nonscattering regime pave the way to a plethora of applications such as nonlinear harmonic generation, enhanced lasing and Raman scattering, and ultrasensitive sensing. On the other hand, we believe that the perfect absorption delivered naturally by low-absorbing dielectric particles could be of a significant relevance for the future device applications that require efficient light trapping and absorption, such as active integrated photonic circuits, selective thermal emitters, or microwave-to-infrared signature controllers.

\section{ACKNOWLEDGMENTS}

The experimental studies of the reflection and transmission coefficients in the microwave frequency range have been supported by the Russian Science Foundation (Grant No. 17-79-20379). A.S. acknowledges the support from the President of the Russian Federation (Grant No. MK-3620.2019.8). Y.S.K. acknowledges the support from the Strategic Fund of the Australian National University. V.R.T. acknowledges the hospitality and financial support of the Jilin University.

H.K.S. and A.S. contributed equally to this work.
[1] M. Kerker, The Scattering of Light (Academic Press, Inc., New York, 1969).

[2] H. C. van de Hulst, Light Scattering by Small Particles (Dover Publications, Inc., New York, 1981).

[3] C. F. Bohren and D. R. Huffman, Absorption and Scattering of Light by Small Particles (Wiley, New York, 1998).

[4] F. Frezza, F. Mangini, and N. Tedeschi, J. Opt. Soc. Am. A 35, 163 (2018).

[5] M. I. Mishchenko, W. J. Wiscombe, J. W. Hovenier, and L. D. Travis, Overview of scattering by nonspherical particles, in Light Scattering by Nonspherical Particles, edited by M. I. Mishchenko, J. W. Hovenier, and L. D. Travis (Academic Press, San Diego, 2000), Chap. 2, pp. 29-60.

[6] F. M. Kahnert, J. Quant. Spectrosc. Radiat. Transfer 79-80, 775 (2003).

[7] T. Wriedt, Mie theory: A review, in The Mie Theory. Springer Series in Optical Sciences, edited by W. Hergert and T. Wriedt (Springer, Berlin, Heidelberg, 2012), Vol. 169, Chap. 2, pp. 5371.

[8] A. V. Osipov and S. A. Tretyakov, Modern Electromagnetic Scattering Theory with Applications (Wiley, Chichester, 2017).

[9] A. B. Evlyukhin, T. Fischer, C. Reinhardt, and B. N. Chichkov, Phys. Rev. B 94, 205434 (2016).

[10] P. D. Terekhov, V. E. Babicheva, K. V. Baryshnikova, A. S. Shalin, A. Karabchevsky, and A. B. Evlyukhin, Phys. Rev. B 99, 045424 (2019).

[11] E. A. Gurvitz, K. S. Ladutenko, P. A. Dergachev, A. B. Evlyukhin, A. E. Miroshnichenko, and A. S. Shalin, Laser Photonics Rev. 13, 1970025 (2019).

[12] R. Alaee, C. Rockstuhl, and I. Fernandez-Corbaton, Adv. Opt. Mater. 7, 1800783 (2019).

[13] R. Rezvani Naraghi, S. Sukhov, and A. Dogariu, Opt. Lett. 40, 585 (2015)

[14] C. Díaz-Avinó, M. Naserpour, and C. J. Zapata-Rodríguez, Opt. Express 24, 18184 (2016).

[15] T. Yamane, A. Nishikata, and Y. Shimizu, IEEE Trans. Electromagn. Compat. 42, 441 (2000).

[16] J. C. Sureau, IEEE Trans. Antennas Propag. 15, 657 (1967).
[17] P. S. Kildal, A. A. Kishk, and A. Tengs, IEEE Trans. Antennas Propag. 44, 1509 (1996).

[18] M. Riso, M. Cuevas, and R. A. Depine, J. Opt. (Bristol, UK) 17, 075001 (2015).

[19] M. Naserpour, C. J. Zapata-Rodríguez, S. M. Vuković, H. Pashaeiadl, and M. R. Belić, Sci. Rep. 7, 12186 (2017).

[20] V. I. Fesenko, V. I. Shcherbinin, and V. R. Tuz, J. Opt. Soc. Am. A 35, 1760 (2018).

[21] V. I. Shcherbinin, V. I. Fesenko, and V. R. Tuz, J. Opt. Soc. Am. B 35, 2066 (2018).

[22] M. Kerker, D.-S. Wang, and C. L. Giles, J. Opt. Soc. Am. 73, 765 (1983).

[23] B. García-Cámara, R. Alcaraz de la Osa, J. M. Saiz, F González, and F. Moreno, Opt. Lett. 36, 728 (2011).

[24] W. Liu and Y. S. Kivshar, Philos. Trans. R. Soc., A 375, 20160317 (2017).

[25] P. D. Terekhov, H. K. Shamkhi, E. A. Gurvitz, K. V. Baryshnikova, A. B. Evlyukhin, A. S. Shalin, and A. Karabchevsky, Opt. Express 27, 10924 (2019).

[26] W. Liu and Y. S. Kivshar, Opt. Express 26, 13085 (2018).

[27] K. V. Baryshnikova, M. I. Petrov, V. E. Babicheva, and P. A. Belov, Sci. Rep. 6, 22136 (2016).

[28] V. E. Babicheva, M. I. Petrov, K. V. Baryshnikova, and P. A. Belov, J. Opt. Soc. Am. B 34, D18 (2017).

[29] M. Decker, I. Staude, M. Falkner, J. Dominguez, D. N. Neshev, I. Brener, T. Pertsch, and Y. S. Kivshar, Adv. Opt. Mater. 3, 813 (2015).

[30] V. E. Babicheva and A. B. Evlyukhin, Laser Photonics Rev. 11, 1700132 (2017).

[31] Z.-J. Yang, R. Jiang, X. Zhuo, Y.-M. Xie, J. Wang, and H.-Q Lin, Phys. Rep. 701, 1 (2017).

[32] H. K. Shamkhi, K. V. Baryshnikova, A. Sayanskiy, P. Kapitanova, P. D. Terekhov, P. Belov, A. Karabchevsky, A. B. Evlyukhin, Y. Kivshar, and A. S. Shalin, Phys. Rev. Lett. 122, 193905 (2019).

[33] S. D. Swiecicki and J. E. Sipe, J. Opt. (Bristol, UK) 19, 095006 (2017).

[34] F. J. García De Abajo, Rev. Mod. Phys. 79, 1267 (2007). 
[35] J. W. Goodman, Introduction to Fourier Optics, 3rd ed., McGraw-Hill Physical and Quantum Electronics Series (W. H. Freeman, Englewood, 2005).

[36] C. Qian, X. Lin, Y. Yang, X. Xiong, H. Wang, E. Li, I. Kaminer, B. Zhang, and H. Chen, Phys. Rev. Lett. 122, 063901 (2019).

[37] I. Staude, A. E. Miroshnichenko, M. Decker, N. T. Fofang, S. Liu, E. Gonzales, J. Dominguez, T. S. Luk, D. N. Neshev, I. Brener, and Y. Kivshar, ACS Nano 7, 7824 (2013).

[38] A. B. Evlyukhin, C. Reinhardt, A. Seidel, B. S. Luk'yanchuk, and B. N. Chichkov, Phys. Rev. B 82, 045404 (2010).

[39] V. A. Markel, J. Phys. B: At., Mol. Opt. Phys. 38, L115 (2005).

[40] W. Liu, A. E. Miroshnichenko, D. N. Neshev, and Y. S. Kivshar, Phys. Rev. B 86, 081407(R) (2012)

[41] C. R. Simovski, A. S. Shalin, P. M. Voroshilov, and P. A. Belov, J. Appl. Phys. 114, 103104 (2013).

[42] V. E. Babicheva and A. B. Evlyukhin, ACS Photonics 5, 2022 (2018).

[43] M. G. Silveirinha, Phys. Rev. A 89, 023813 (2014).

[44] M. V. Rybin, K. L. Koshelev, Z. F. Sadrieva, K. B. Samusev, A. A. Bogdanov, M. F. Limonov, and Y. S. Kivshar, Phys. Rev. Lett. 119, 243901 (2017).
[45] V. E. Babicheva, MRS Commun. 8, 1455 (2018).

[46] A. Sayanskiy, A. S. Kupriianov, S. Xu, P. Kapitanova, V. Dmitriev, V. V. Khardikov, and V. R. Tuz, Phys. Rev. B 99, 085306 (2019).

[47] S. Xu, A. Sayanskiy, A. S. Kupriianov, V. R. Tuz, P. Kapitanova, H.-B. Sun, W. Han, and Y. S. Kivshar, Adv. Opt. Mater. 7, 1801166 (2019).

[48] P. Lalanne, W. Yan, K. Vynck, C. Sauvan, and J. P. Hugonin, Laser Photonics Rev. 12, 1700113 (2018).

[49] N. Ismail, C. C. Kores, D. Geskus, and M. Pollnau, Opt. Express 24, 16366 (2016).

[50] M. Born and E. Wolf, Principles of Optics: Electromagnetic Theory of Propagation, Interference and Diffraction of Light, 7th ed. (Cambridge University Press, Cambridge, 1999).

[51] A. E. Miroshnichenko, A. B. Evlyukhin, Y. S. Kivshar, and B. N. Chichkov, ACS Photonics 2, 1423 (2015).

[52] D. A. Baranov, P. A. Dmitriev, I. S. Mukhin, A. K. Samusev, P. A. Belov, C. R. Simovski, and A. S. Shalin, Appl. Phys. Lett. 106, 171913 (2015).

[53] A. S. Shalin, JETP Lett. 91, 636 (2010).

[54] A. S. Shalin and S. G. Moiseev, Opt. Spectrosc. 106, 916 (2009). 